\title{
Left Atrial Multi-planar ICE Imaging for the Guidance of Left Atrial Appendage Closure: A Comparative Study with TEE Imaging
}

Fangyi Xiao ${ }^{1}$, yanyan chen ${ }^{2}$, Yat Lam $^{3}$, yi he chen ${ }^{4}$, Liangguo Wang ${ }^{5}$, Ying Fang ${ }^{2}$, Lan $\mathrm{Su}^{6}$, and weijian huang ${ }^{7}$

${ }^{1}$ the First Affiliated Hospital of Wenzhou Medical University ZheJiang 325000 P.R. China

${ }^{2}$ The First Afliated Hospital of Wenzhou Medical University

${ }^{3}$ Central Medical Limited

${ }^{4}$ the second afliated hospital of Wenzhou Medical University

${ }^{5}$ he First Affiliated Hospital of Wenzhou Medical University

${ }^{6}$ The First Affiliated Hospital of Wenzhou Medical University

${ }^{7}$ The First Affiliated Hospital of Wenzhou Medical College

October 9, 2020

\begin{abstract}
Introduction: Transesophageal echocardiography (TEE) fails to display optimal views to guide left atrial appendage closure (LAAC) procedure in some patients due to atrio-esophageal anatomical limitation. We aimed to investigate if intracardiac echocardiography (ICE) conducted from left atrium (LA) achieves comparable multi-planar views and clinical outcomes as TEE during LAAC. Methods and Results: This study prospectively enrolled 102 consecutive patients with non-valvular atrial fibrillation receiving LAmbre implants under local anesthesia at the First Affiliated Hospital of Wenzhou Medical University from August 2018 to July 2019. The procedures were guided by either ICE ( $\mathrm{n}=40)$, TEE ( $\mathrm{n}=50)$ or combined ICE-TEE ( $\mathrm{n}=12)$. A novel multi-angled "FLAVOR" approach was used in ICE group for assessment. ICE allowed visualization of implanted device in all patients at all proposed angles with long-axis views, while TEE failed to do so in at least one of the angles in $36 \%$ of cases. In the combined ICE-TEE cohort, TEE failed peri-device leak assessment in 2 patients. Rates of procedural success, recapture, resizing and complications were similar between ICE and TEE groups. Fluoroscopy time, radiation dose and volume of contrast use in ICE group were significantly lower than the TEE cohort. At 45-day TEE follow-up, rate and degree of peri-device leaks were similar between the ICE and TEE groups. Conclusions: A systematic approach using ICE to guide LAmbre LAA occlusion was safe and feasible. This method was more reliable in comprehensive, multi-angled imaging assessment, and achieving shorter fluoroscopy time, lower radiation dose and less contrast use than TEE.
\end{abstract}

\section{TITLE PAGE}

Left Atrial Multi-planar ICE Imaging for the Guidance of Left Atrial Appendage Closure: A Comparative Study with TEE Imaging

\section{Authors and affiliation}

Fangyi Xiao $\mathrm{MD}^{1,2+}$, Yanyan Chen $\mathrm{MD}^{3+}$, Yat-Yin Lam $\mathrm{MD}^{4}$, Yihe Chen $\mathrm{MD}^{1,2}$, Liangguo Wang $\mathrm{MD}^{1,2}$, Ying Fang $\mathrm{MD}^{1,2}$, Lan Su MD ${ }^{1,2}$, Weijian Huang $\mathrm{MD}^{1,2} *$

1. Department of Cardiology, the First Affiliated Hospital of Wenzhou Medical University, Nanbaixiang, Wenzhou 325000, P.R. China. 
2. the Key Lab of Cardiovascular Disease of Wenzhou, Nanbaixiang, Wenzhou 325000, P.R. China.

3. Department of Neurology, the First Affiliated Hospital of Wenzhou Medical University, Nanbaixiang, Wenzhou 325000, P.R. China.

4. Hong Kong Asia Heart Center, Canossa Hospital, 1 Old Peak Road, Hong Kong

+ The first two authors contributed equally to the study.

*Corresponding author: Weijian Huang, MD; Department of Cardiology, The First Affiliated Hospital of Wenzhou Medical University; the Key Lab of Cardiovascular Disease of Wenzhou, Nanbaixiang, Wenzhou 325000, P.R. China. E-mail:weijianhuang69@126.com;

Funding: This work was supported by Key Research and Development Program of Zhejiang (2019C03012), Major Project of the Science and Technology of Wenzhou (ZS2017010) and Project of the Science and Technology of Wenzhou (Y20180647)

Disclosures: None

\begin{abstract}
Introduction: Transesophageal echocardiography (TEE) fails to display optimal views to guide left atrial appendage closure (LAAC) procedure in some patients due to atrio-esophageal anatomical limitation. We aimed to investigate if intracardiac echocardiography (ICE) conducted from left atrium (LA) achieves comparable multi-planar views and clinical outcomes as TEE during LAAC.

Methods and Results: This study prospectively enrolled 102 consecutive patients with non-valvular atrial fibrillation receiving LAmbre implants under local anesthesia at the First Affiliated Hospital of Wenzhou Medical University from August 2018 to July 2019. The procedures were guided by either ICE (n=40), TEE $(\mathrm{n}=50)$ or combined ICE-TEE $(\mathrm{n}=12)$. A novel multi-angled "FLAVOR" approach was used in ICE group for assessment. ICE allowed visualization of implanted device in all patients at all proposed angles with longaxis views, while TEE failed to do so in at least one of the angles in 36\% of cases. In the combined ICE-TEE cohort, TEE failed peri-device leak assessment in 2 patients. Rates of procedural success, recapture, resizing and complications were similar between ICE and TEE groups. Fluoroscopy time, radiation dose and volume of contrast use in ICE group were significantly lower than the TEE cohort. At 45-day TEE follow-up, rate and degree of peri-device leaks were similar between the ICE and TEE groups.
\end{abstract}

Conclusions: A systematic approach using ICE to guide LAmbre LAA occlusion was safe and feasible. This method was more reliable in comprehensive, multi-angled imaging assessment, and achieving shorter fluoroscopy time, lower radiation dose and less contrast use than TEE.

Keywords: intracardiac echocardiography; left atrial appendage closure; transesophageal echocardiography; atrial fibrillation

\title{
1 Introduction
}

Transcatheter left atrial appendage closure (LAAC) has become a reasonable alternative to long term oral anticoagulant therapy (OAT) to prevent stroke in patients with non-valvular atrial fibrillation (NVAF) ${ }^{1}$. The procedure is predominantly guided by transesophageal echocardiography (TEE) under general anesthesia or deep sedation. In many centers in China, it is performed under local anesthesia instead. The occluder is first implanted with fluoroscopic guidance, and the TEE probe is then placed with local laryngeal anesthesia to assess the device position and residual peri-device leak before final device release. TEE examination is generally safe and widely used in LAAC procedure, but it remains limited by requirement of general anesthesia or deep sedation and small risk of severe esophageal complications. More importantly, TEE fails to optimally visualize left atrial appendage (LAA) structures and devices especially at long-axis view in some patients due to varied anatomical positions of the esophagus and the LAA. Intracardiac echocardiography (ICE) has been reported as an alternative to TEE for guiding LAAC ${ }^{2-6}$, however, the feasibility of achieving consistent multi-planar imaging quality remains unproven. Therefore, we aimed to investigate whether ICE could achieve comparable multi-angled views as TEE during LAAC. 


\section{Methods}

\subsection{Study design and patient population}

All consecutive NVAF patients at high risk of stroke (CHA2DS2-VASc scores[?]2) underwent LAmbre (Lifetech Scientific, Shenzhen, China) occluders implantation at the First Affiliated Hospital of Wenzhou Medical University from August 2018 to July 2019 were enrolled in this study. All the procedures were performed under local anesthesia. There were 40 cases in the ICE group, including 23 cases with combined catheter ablation and LAAC in a single operation. There were 50 cases in the TEE group, including 28 cases with combined procedures. Another 12 patients underwent LAAC with both ICE and TEE imaging. All patients received pre-procedural TEE to assess LAA anatomy and to exclude LAA thrombus. Written informed consent was obtained from all the participants and the study was approved by ethical committee of the local institution.

\subsection{LAAC with ICE imaging}

LAAC with ICE imaging was performed under local anesthesia without conscious sedation. Double right femoral vein access was obtained with an $11 \mathrm{~F}$ 20-cm Terumo sheath (Terumo Europe, Leuven, Belgium) for introducing the 10F ICE catheter (Sound-Star, Biosense Webster, Diamond Bar, CA) and another 8.5F Swartz long sheath (SL1; AF Division, SJM, Minneapolis, MN). The geometry map of the left atrium (LA) including LAA was created by ICE probe manipulation at the right median atrial septum and at the coronary sinus ostium under CARTO3 three-dimensional mapping system (Biosense Webster, Inc). Shortly after successful ICE-guided transseptal puncture with a needle (BRK, SJM), a $6 \mathrm{~F}$ pigtail catheter was advanced to the LAA to perform LAA angiography, with LA pressure[?]12 $\mathrm{mmHg}$, at right anterior oblique and caudal projections. The angiographic diameters of LAA ostium and landing zone then were measured. After the pigtail catheter was removed, a guidewire was inserted through Swartz sheath to the left superior pulmonary vein (LSPV) under the ICE guidance. The Swartz sheath was further exchanged with a $10 \mathrm{~F}$ delivery sheath to pre-dilate the transseptal puncture site. Then the delivery sheath was retracted into the inferior vena cava to allow the ICE catheter advance into LA through the same site which was tagged on the CARTO-Sound image guided by CARTO3 system without the need of any fluoroscopic guidance. The echocardiographic LAA ostium and landing zone diameters were measured using the "FLAVOR (F our L ong- $\mathbf{A}$ xis $\mathbf{V}$ iews a round $\mathbf{O r}$ ifice)" approach at $90^{\circ}, 135^{\circ}, 0^{\circ}$ and $45^{\circ}$ (Fig. 1 and 2 ). The $10 \mathrm{~F}$ delivery sheath was advanced again to the LA over the guidewire placed in LSPV through the same transseptal puncture site with caution. The appropriate size of LAmbre device was selected based on both angiographic and echocardiographic measurements. Guided by fluoroscopy and ICE, the umbrella of the device was fully deployed at the landing zone by pushing the delivery cable, and the cover of the device was then deployed at the LAA ostium by unsheathing the delivery catheter. The position of the device and the degree of residual flow were assessed with ICE (Fig. 2). The device was released when the COST criteria (including Umbrella deployed beyond $\mathbf{C}$ ircumflex artery; Umbrella was fully $\mathbf{O}$ pen; Peri-device optimal $\mathbf{S}$ ealing (leak [?] 3mm); Device stability confirmed by $\mathbf{T}$ ug test) were met and the cover of the device had no impingement on mitral valve and/or pulmonary vein ostium. Otherwise, recapture and/or resizing of the device would be attempted. Additional ICE assessment with " FLAVOR " approach would be performed after release of the device.

\section{3 "FLAVOR" (F our $\mathbf{L}$ ong-A xis $\mathbf{V}$ iews around Or ifice) approach}

The constructed LA geometry map at the antero-posterior projection was adjusted rightward and upward until LAA orifice was adequately exposed. The ICE probe was manipulated with caution to achieve the proposed 4 imaging planes (the angle between adjacent imaging planes was approximately 45deg) for comprehensive evaluation of the LAA or device. Each plane was adjusted to show the long-axis view of LAA or the device. The ICE probe was initially advanced to the top of LA with slight posterior flexion to obtain the first view (similar to the TEE 90deg view), which we defined as the 90deg view. The second view (similar to the TEE 45deg view) could be achieved through right and ante-flexion (R+A flex) of the ICE probe. The ICE probe was usually placed outside the left upper pulmonary vein ostium, and we defined as the $135 \mathrm{deg}$ view. Next, the ICE probe was usually positioned between the left upper and lower pulmonary veins on the 
LA posterior wall to form the third view (similar to the TEE 0deg view) by further rightward flexion (R flex), and we defined as the 0deg view. Finally, the ICE catheter was rotated anticlockwise and advanced to mitral isthmus or across the mitral valve with posterior flexion to form the fourth view (similar to the TEE 135deg view), which we defined as the $45 \mathrm{deg}$ view. This systematic approach allowed comprehensive evaluation of LAA or devices mimicking TEE echo windows (Fig. 1 and 2).

\subsection{LAAC with TEE imaging}

LAAC with TEE imaging also was performed under local anesthesia without conscious sedation. Transseptal puncture was performed with the $8.5 \mathrm{~F}$ Swartz long sheath and transseptal puncture needle placed via right femoral venous access under fluoroscopic guidance. Then, a $6 \mathrm{~F}$ pigtail catheter was inserted along the Swartz sheath for LAA angiography at the right anterior oblique and caudal projections. The sizes of the landing zone and the orifice were measured. The appropriate device size was selected according to the intraoperative angiographic and preoperative echocardiographic measurements and the device was implanted as aforementioned. Multi-planar TEE imaging $(0 \mathrm{o}, 45 \mathrm{o}, 90 \mathrm{o}$ and 135o) under local laryngeal anesthesia with tetracaine were performed until no significant peri-device leak was detected by multi-projection cineangiography and the stability was confirmed by tag test. The device would only be released when COST criteria were met. TEE exams were performed by two experienced echocardiographers.

\subsection{LAAC with simultaneous TEE and ICE imaging}

Under local anesthesia, the LAmbre device was deployed under fluoroscopic guidance. TEE exams followed by ICE exam with "FLAVOR" approach were subsequently performed. The outcomes of the 2 imaging modality were compared.

\subsection{Radiofrequency ablation for atrial fibrillation}

In patients with combined procedures, radiofrequency ablation was performed prior to LAAC. An additional decapolar catheter was placed in the coronary vein through the left femoral vein access in patients with combined procedure. In ICE group, ICE was used to create a LA anatomical map in the right atrium. Single transseptal puncture was guided by ICE. An ablation catheter (Thermocool SmartTouch, Biosense Webster, Inc) supported by a steerable sheath (Agilis NXT; St. Jude Medical, St Paul, MN, USA) was used to perform ablation index guided circumferential pulmonary vein isolation. In patients with persistent atrial fibrillation, cardioversions were delivered to restore sinus rhythm after CPVI. Substrate mapping and ablation then was performed as previously described ${ }^{7}$. In TEE group, following double transseptal punctures, the LA anatomical map was created using a 10-pole spiral catheter (Lasso NAV, Biosense Webster, Inc.) under Carto3 mapping system guidance. The ablation strategy was similar in the ICE group.

\subsection{Post-procedural management and follow-up}

All patients underwent transthoracic echocardiography to rule out pericardial effusion the following day. OAT was administered for at least 45 days and 2 months in patients with isolated LAAC and those with combined procedures, respectively. This was followed by aspirin and clopidogrel for 6 months and aspirin or clopidogrel lifelong. At 45-day, 6-month and 12-month follow-up, TEE was performed to evaluate the device position, residual flow and thrombus formation on the device surface. Routine electrocardiogram and $24 \mathrm{~h}$ Holter monitoring were examined at 3 and 6 -months post procedure, and 7 -day Holter monitoring was performed at 12 months post procedure.

\subsection{Statistical analysis}

Quantitative data were expressed as mean +- SD and were compared using 2-tailed t test. Categorical data were expressed as percentages and compared using the Chi square test. $\mathrm{P}<0.05$ was considered statistically significant.

\section{Results}

\subsection{Baseline characteristics of the study subjects}


Table 1 shows the baseline demographic data of ICE and TEE groups. The mean age was 72 and 69 years old and the prevalence of paroxysmal atrial fibrillation was 35\% and $20 \%$ in the ICE and TEE groups, respectively. The $\mathrm{CHA}_{2} \mathrm{DS}_{2}$-VASc scores were similar $(4.2+-1.5$ and $4.1+-1.3)$ in 2 groups. Frequency of history of prior stroke/TIA was $52.5 \%$ and $64 \%$, respectively. The HAS-BLED score was significantly higher in the TEE group.

\subsection{Images obtained by the "FLAVOR" approach comparing with those by TEE}

The "FLAVOR" approach clearly displayed the long-axis views of the device at 4 angles in all cases in ICE group (Fig. 2). However, 18 (36\%) cases in TEE group failed to show long-axis views including short-axis view or unsatisfactory long-axis view (a view between long-axis and short-axis) (Fig. 3) of the device at 1 of the 4 angles, including 17 cases (34\%) at 135deg, 1 case (2\%) at 0deg (Table 2). Among 12 patients in whom both ICE and TEE were used during LAAC, TEE failed to display long-axis views at 135deg in 4 patients (Fig. 4). In 2 of the 4 cases, significant residual flow was detected by ICE instead of TEE (Supplementary movie 1).

\subsection{Procedural characteristics and follow-up data}

All procedures were successful. Most patients had complete LAA seal except for 2 cases in ICE group and 3 cases in TEE group with small residual leak $<3 \mathrm{~mm}$. The fluoroscopy time, radiation dose and contrast usage were significantly lower in ICE group than TEE group. There was no significant difference in procedural time and device resizing rate (Table 2). The 45-day TEE follow-up rates were similar in both groups $(95 \%$ vs. 94\%). The complete seal rate in ICE group and TEE group was $68.4 \%$ and $70.2 \%$, respectively. No peri-device leak $>3 \mathrm{~mm}$ was observed during follow-up TEE exams. Compared with TEE group, ICE group had a higher trend in prevalence of iatrogenic atrial septal defects $(52.6 \% \mathrm{vs} 34 \%, \mathrm{P}=0.085)$, but the sizes were similar in both groups ( $3.6 \mathrm{~mm}$ vs $3.3 \mathrm{~mm}, \mathrm{P}=0.529$ ) (Table 3 ). No device-related thrombus was detected in either group.

\subsection{Adverse events}

One patient in TEE group had an ischemic stroke 2 days after the procedure. Severe stenosis basilar artery was found as the cause of the stroke event. One patient in TEE group who received combined procedure developed delayed pericardial effusion, requiring pericardiocentesis at $18^{\text {th }}$ day after the operation. Minor complication including a pseudoaneurysm at left femoral puncture site in 1 patient with combined treatment in ICE group. No other serious complication was observed during 2 months follow-up.

\section{Discussion}

\subsection{Main findings}

This study is the first to our knowledge to demonstrate the safety and feasibility of a systematic ICE protocol ("FLAVOR" approach) in achieving consistent, high quality, multi-planar echocardiographic imaging for guiding LAAC under local anesthesia. This novel approach did not compromise patients' peri-procedural and short-term outcomes and it collected better comprehensive multi-angled imaging information than TEE with additional advantages of less radiation and contrast use.

\subsection{ICE images for evaluation of LAAC}

TEE has long been the standard for guiding LAAC. It can exclude LAA thrombus during the operation, guiding transseptal puncture, facilitating placement of pigtail, sheath and device, and assessing the device position and residual flow. Precise assessment of implanted device position and residual flow was commonly based on optimal long-axis views of the device displayed by TEE at different imaging planes. However, limited by the varied anatomical relationship between the esophagus and the LAA, the long-axis images of the LAA and implanted device in some patients cannot be displayed satisfactorily, mostly at 135deg, which impacts the accuracy in assessing LAA sizing, device position and residual leakage. The use of ICE has been reported to be able to obtain sufficient images as TEE to guide LAAC with similar effectiveness and safety ${ }^{5,6,8,9}$. In the early studies, ICE probe was placed in the right atrium, coronary sinus, right ventricular outflow tract, 
or pulmonary artery to guide $\mathrm{LAAC}^{2,4,8}$. However, ICE examination conducted in the right atrium failed to show consistent optimal images from LA/LAA because of the inherent far-field limitation. The LAA images obtained with the ICE probe positioned in LA were dramatically improved ${ }^{3,5,6,9-11}$. However, the systematic approach for manipulating ICE probe to achieve consistent, comparable multi-angled images as TEE exam was not well described. Frangieh et $\mathrm{al}^{9}$ and Korsholm et $\mathrm{al}^{5}$ did not discuss the detailed approach to navigate ICE probe in LAAC. Kim et $\mathrm{al}^{10}$ placed the ICE probe at a single position in LA to guide LAAC which might affect assessment accuracy. Masson et al and Alkhouli et al ${ }^{3,12}$ reported using 2 ICE views guide LAAC while Hemam et $\mathrm{al}^{6}$ went further to show that ICE could be placed in 4 different positions including the top of LA, the left upper and lower pulmonary veins, and through the mitral valve to better evaluate the LAA and the device. But these approaches, without using the LA geometry map described by us, were limited by the varied anatomical relationships among LAA, pulmonary veins and mitral valves and the technical difficulty in acquiring reproducible, comparable and omni-plane views similar to those obtained by TEE counterparts.

TEE allows to assess the LAA and the device from a full 0-180deg sweep of imaging probe. To compare the performance of ICE versus TEE, we proposed the step-by-step "FLAVOR" approach in this study which consistently obtained high quality, reproducible images at 4 proposed imaging angles. In our study, all the proposed views could be obtained with good quality in ICE group. The acquisition of images was independent of esophageal-LAA anatomical relation which clearly limits the TEE performance, with more than one-third cases in TEE group were unable to display the long-axis image at one of the four angles, mostly at 135deg. We suggest that with proper training on the "FLAVOR" approach, intra-LA ICE imaging could be an attractive alternative for guiding LAAC. While the cost of ICE catheter remains a barrier limiting its wide application, it would be appealing for patients undergoing combined AF ablation and LAAC because the catheter would be needed for transseptal punctures, LA geometry mapping and guiding ablation anyway.

\subsection{The effectiveness of LAAC with ICE imaging}

Previous studies ${ }^{5,6,10}$ compared the ICE-guided LAAC under local anesthesia with the TEE-guided LAAC under general anesthesia, and found that both of them had a very high procedural success rate. The application of ICE did not reduce the success rate, nor did it increase device recapture times or resizing rate. Our results are consistent with these findings, showing LAAC success rate of $100 \%$ for both approaches. Intra-operative residual leak $<3 \mathrm{~mm}$ was observed in 2 cases in the ICE group and 3 cases in the TEE group after device release. The device recapture and resizing rates were similar in the 2 groups. Whether the use of ICE has a favorable impact on total procedural time, radiation time, radiation dose and contrast agent dose remained controversial ${ }^{5,6,9,10}$. This is largely attributed to different workflows during the procedure. In contrast to previous studies, we performed all the procedures under local anesthesia. We conducted a brief TEE examination only at the end of the procedure to verify the device position and the degree of residual leak. In ICE group, we used the LA geometry map to facilitate ICE catheter manipulation to obtain the optimal imaging plane thereby sparing the operator the need for intense fluoroscopy control. This approach leads to reduced fluoroscopy time and radiation exposure without a increase in procedure time in ICE cohort.

\subsection{The safety of LAAC with ICE imaging}

No severe intra-procedural complications occurred in either group. There was no device-related thrombus, device embolism, major bleeding or death occurred in either group at 2 months follow-up. One patient in TEE group developed pericardial effusion which was inherent to the procedure. No complication was related to the manipulation of ICE catheter.

The presence of residual iatrogenic atrial septal defect (iASD) after transseptal catheterization is not uncommon. Sheldon et $\mathrm{al}^{13}$ reported that patients undergoing LAAC with WATCHMAN device as a part of PROTECT-AF study had a high incidence of $34 \%$ at 45 days TEE follow-up. However, the study also showed a high spontaneous closure rate (7\% at 12 months) of iASDs that was not associated with an increased rate of stroke/systemic embolization during long-term follow-up. Chan et $\mathrm{al}^{14}$ documented an occurrence rate for persistent iASD of 30.6\% at 9-month and 20\% at 6-year TEE follow-up in AF patients with cryoballoon 
pulmonary vein isolation. Two patients with a defect size of over $10 \mathrm{~mm}$ required percutaneous closure due to significant left-to-right shunting in that study. The long-term effect of persistent iASD on echocardiographic parameters and on clinical outcomes remain unclear. Korsholm et $\mathrm{al}^{5}$ compared ICE-guided LAAC under local anesthesia with TEE-guided LAAC under general anesthesia using Amplatzer Cardiac Plug or Amulet device. The mean 55-day follow-up of TEE after procedure showed no significant difference in incidence (35\% vs $26 \%$ ) and size of iASD between the two groups. Our study showed that the ICE group had numerically more iASD cases than the TEE group without increased size at 45-day follow-up. Whether ASD would close spontaneously remains to be assessed at long-term follow-up.

\subsection{Study limitations}

This was a small, non-randomized feasibility study. Although the study results could not be fully extrapolated to other LAA occluding devices, but were more representative in pacifier LAAC devices. Three-dimensional (3D) TEE with multi-planar reconstruction function may help to enhance the accuracy of LAA and device assessment but the precision of 3D images still based on the quality of $2 \mathrm{D}$ images ${ }^{15,16}$. The development of 3D-ICE is currently underway which may provide new insights in guiding LAAC in the future ${ }^{17}$. Our study only used a single type of ICE catheter (Sound-Star, Biosense Webster, Diamond Bar, CA) which was soft and flexible allowing safe manipulation in LA under Carto3 mapping system guidance. Lastly, ICE catheter was maneuvered by experienced electrophysiologists in current study.

\section{Conclusions}

ICE-guided LAAC with the "FLAVOR" approach is safe, feasible and is associated with reduced fluoroscopy time, radiation dose and contrast usage without compromising procedure time and outcomes as compared to TEE counterparts.

\section{Acknowledgements}

None.

\section{Reference}

1 Glikson M, Wolff R, Hindricks G, Mandrola J, Camm AJ, Lip GYH, et al. EHRA/EAPCI expert consensus statement on catheter-based left atrial appendage occlusion - an update. Europace. 2019.

2 Ho IC, Neuzil P, Mraz T, Beldova Z, Gross D, Formanek P, et al. Use of intracardiac echocardiography to guide implantation of a left atrial appendage occlusion device (PLAATO). Heart rhythm. 2007;4(5):567-71.

3 Masson JB, Kouz R, Riahi M, Nguyen Thanh HK, Potvin J, Naim C, et al. Transcatheter Left Atrial Appendage Closure Using Intracardiac Echocardiographic Guidance From the Left Atrium. Can J Cardiol. 2015;31(12):1497 e7- e14.

4 Matsuo Y, Neuzil P, Petru J, Chovanec M, Janotka M, Choudry S, et al. Left Atrial Appendage Closure Under Intracardiac Echocardiographic Guidance: Feasibility and Comparison With Transesophageal Echocardiography. J Am Heart Assoc. 2016;5(10).

5 Korsholm K, Jensen JM, Nielsen-Kudsk JE. Intracardiac Echocardiography From the Left Atrium for Procedural Guidance of Transcatheter Left Atrial Appendage Occlusion. JACC Cardiovasc Interv. 2017;10(21):2198-206.

6 Hemam ME, Kuroki K, Schurmann PA, Dave AS, Rodriguez DA, Saenz LC, et al. Left atrial appendage closure with the Watchman device using intracardiac vs transesophageal echocardiography: Procedural and cost considerations. Heart rhythm : the official journal of the Heart Rhythm Society. 2019;16(3):334-42.

7 Yang B, Jiang C, Lin Y, Yang G, Chu H, Cai H, et al. STABLE-SR (Electrophysiological Substrate Ablation in the Left Atrium During Sinus Rhythm) for the Treatment of Nonparoxysmal Atrial Fibrillation: A Prospective, Multicenter Randomized Clinical Trial. Circ Arrhythm Electrophysiol. 2017;10(11). 
8 Berti S, Paradossi U, Meucci F, Trianni G, Tzikas A, Rezzaghi M, et al. Periprocedural intracardiac echocardiography for left atrial appendage closure: a dual-center experience. JACC Cardiovasc Interv. 2014;7(9):1036-44.

9 Frangieh AH, Alibegovic J, Templin C, Gaemperli O, Obeid S, Manka R, et al. Intracardiac versus transesophageal echocardiography for left atrial appendage occlusion with watchman. Catheter Cardiovasc Interv. 2017;90(2):331-8.

10 Kim DY, Shin SY, Kim JS, Kim SH, Kim YH, Lim HE. Feasibility of intracardiac echocardiography imaging from the left superior pulmonary vein for left atrial appendage occlusion. Int J Cardiovasc Imaging. 2018;34(10):1571-9.

11 Fassini G, Dello Russo A, Conti S, Tondo C. An alternative transseptal intracardiac echocardiography strategy to guide left atrial appendage closure: the first described case. J Cardiovasc Electrophysiol. 2014;25(11):1269-71.

12 Alkhouli M, Chaker Z, Alqahtani F, Raslan S, Raybuck B. Outcomes of Routine Intracardiac Echocardiography to Guide Left Atrial Appendage Occlusion. JACC Clin Electrophysiol. 2020 Apr;6(4):393-400.

13 Singh SM, Douglas PS, Reddy VY. The incidence and long-term clinical outcome of iatrogenic atrial septal defects secondary to transseptal catheterization with a $12 \mathrm{~F}$ transseptal sheath. Circ Arrhythm Electrophysiol. 2011;4(2):166-71.

14 Chan NY, Choy CC, Yuen HC, Chow HF, Fong HF. A Very Long-term Longitudinal Study on the Evolution and Clinical Outcomes of Persistent Iatrogenic Atrial Septal Defect After Cryoballoon Ablation. Can J Cardiol. 2019;35(4):396-404.

15 Al-Kassou B, Tzikas A, Stock F, Neikes F, Volz A, Omran H. A comparison of two-dimensional and real-time 3D transoesophageal echocardiography and angiography for assessing the left atrial appendage anatomy for sizing a left atrial appendage occlusion system: impact of volume loading. EuroIntervention. 2017;12(17):2083-91.

16 Zhou Q, Song H, Zhang L, Deng Q, Chen J, Hu B, et al. Roles of real-time three-dimensional transesophageal echocardiography in peri-operation of transcatheter left atrial appendage closure. Medicine (Baltimore). 2017;96(4):e5637.

17 Enriquez A, Saenz LC, Rosso R, Silvestry FE, Callans D, Marchlinski FE, et al. Use of Intracardiac Echocardiography in Interventional Cardiology: Working With the Anatomy Rather Than Fighting It. Circulation. 2018;137(21):2278-94.
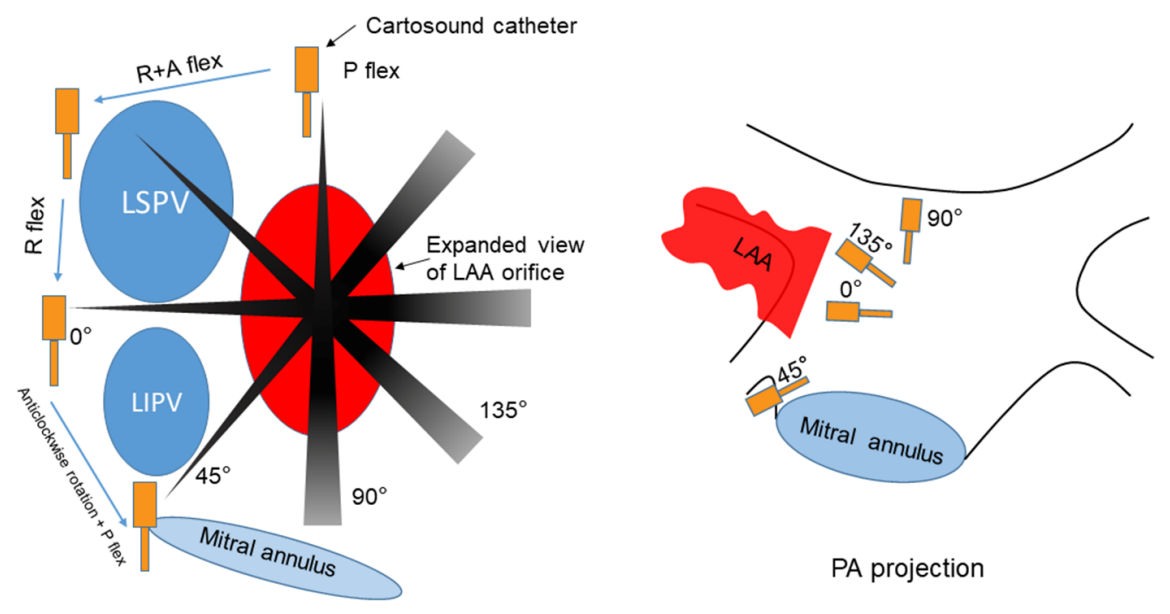

PA projection 
Figure 1 Step by step illustration of "FLAVOR" approach. LAA= left atrial appendage, $\mathrm{P}=$ posterior, $\mathrm{R}=$ right, $\mathrm{A}=$ anterior, $\mathrm{PA}=$ postero-anterior, $\mathrm{LSPV}=$ left superior pulmonary vein, $\mathrm{LIPV}=$ left inferior pulmonary vein.

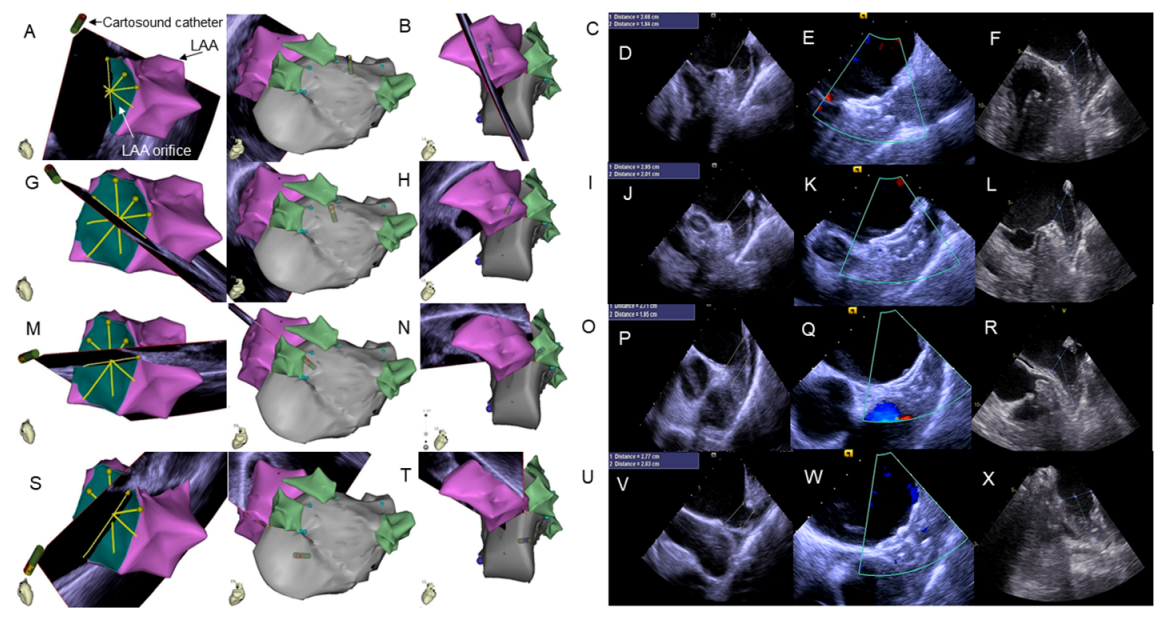

Figure 2 Illustration of ICE with "FLAVOR" approach to measure of LAA orifice and landing zone and to assess of the position of the device and residual flow at four angles $\left(90^{\circ}, 135^{\circ}, 0^{\circ}\right.$, $\left.45^{\circ}\right)$. Expanded view of LAA orifice and ICE planes at $90^{\circ}(\mathrm{A}), 135^{\circ}(\mathrm{G}), 0^{\circ}(\mathrm{M}), 45^{\circ}(\mathrm{S})$; Postero-anterior $(\mathrm{B}$, $\mathrm{H}, \mathrm{N}, \mathrm{T})$ and left lateral views $(\mathrm{C}, \mathrm{I}, \mathrm{O}, \mathrm{U})$ of $3 \mathrm{D}$ reconstruction of the LA and the corresponding position of ICE catheter was showed; measurement of LAA orifice and landing zone $(\mathrm{D}, \mathrm{J}, \mathrm{P}, \mathrm{V})$; and assessment of the device position and residual flow $(\mathrm{E}, \mathrm{K}, \mathrm{Q}, \mathrm{W})$ using ICE with long-axis views at the 4 angles after release. Pre-procedural TEE views of LAA at $90^{\circ}, 45^{\circ}, 0^{\circ}, 135^{\circ}$ (F, L, R, X) were similar to ICE views at $90^{\circ}, 135^{\circ}$, $0^{\circ}, 45^{\circ}(\mathrm{D}, \mathrm{J}, \mathrm{P}, \mathrm{V}) . \mathrm{LAA}=$ left atrial appendage.
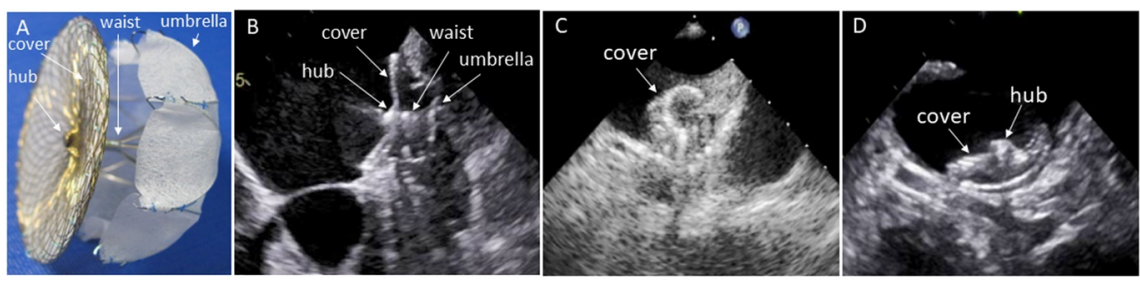

Figure 3 Different views of the LAmbre device. The LAmbre device is a self-expanding nitinolbased device consisting of a fabric-enriched cover and an umbrella connected with a short central waist (A). Illustration of the long-axis view (B), short-axis view (C) and unsatisfactory long-axis view (D) of the device by TEE. 

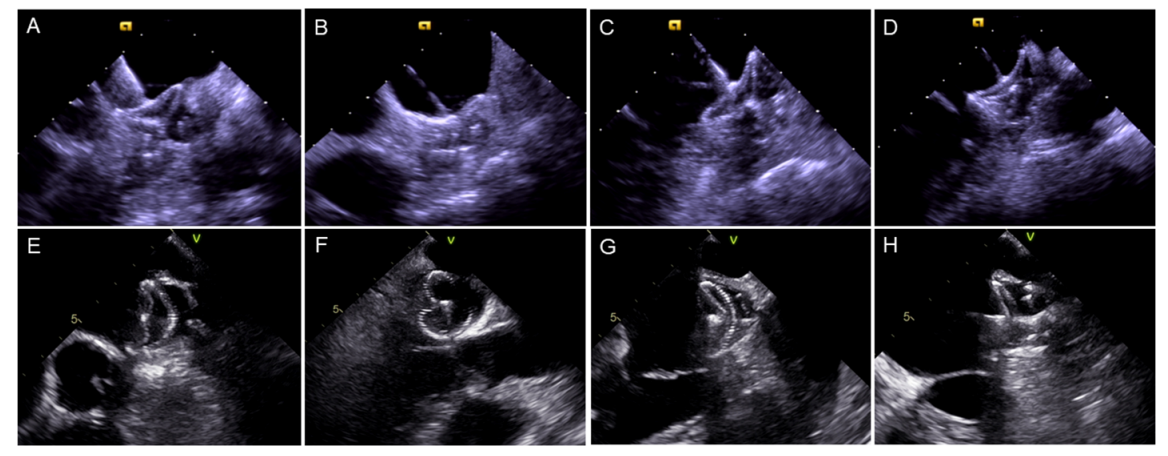

Figure 4 A comparison of ICE views with TEE views at four angles side by side in the assessment of device position before release in the same patient. ICE successfully displayed the long-axis views of the LAmbre device at $0^{\circ}(\mathrm{A}), 45^{\circ}(\mathrm{B}), 90^{\circ}(\mathrm{C}), 135^{\circ}(\mathrm{D})$. TEE showed the long-axis views of the LAmbre device at $0^{\circ}(\mathrm{E}), 90^{\circ}(\mathrm{G}), 45^{\circ}(\mathrm{H})$, but failed at the $135^{\circ}(\mathrm{F})$ and showed the short-axis view of the device.

Supplementary movie 1 Significant residual flow was detected by ICE instead of TEE. TEE was able to display long-axis views of the device at $0^{\circ}(\mathrm{A}), 45^{\circ}(\mathrm{B})$ and $90^{\circ}(\mathrm{C})$ except $135^{\circ}(\mathrm{D})$. No significant residual flow was detected by TEE. Significant residual flow was detected by ICE at $45^{\circ}$ view using "FLAVOR" approach (E, F) immediately after TEE assessment (white arrows). Additional three-dimensional TEE (G) was used to confirm the residual flow in this patient, significant residual flow was visualized (white arrow).

\begin{tabular}{|c|c|c|c|}
\hline $\begin{array}{l}\text { Table } 1 \text { Baseline } \\
\text { characteristics by study } \\
\text { groups }\end{array}$ & $\begin{array}{l}\text { Table } 1 \text { Baseline } \\
\text { characteristics by study } \\
\text { groups }\end{array}$ & $\begin{array}{l}\text { Table } 1 \text { Baseline } \\
\text { characteristics by study } \\
\text { groups }\end{array}$ & $\begin{array}{l}\text { Table } 1 \text { Baseline } \\
\text { characteristics by study } \\
\text { groups }\end{array}$ \\
\hline & $\operatorname{ICE}(\mathrm{n}=40)$ & $\operatorname{TEE}(\mathrm{n}=50)$ & $P$ value \\
\hline Female & $16(40)$ & $13(26)$ & 0.158 \\
\hline Age, yrs & $71.8 \pm 7.1$ & $69.4 \pm 8.4$ & 0.152 \\
\hline BMI & $24.2 \pm 3.1$ & $24.6 \pm 2.6$ & 0.542 \\
\hline PAF & $14(35)$ & $10(20)$ & 0.110 \\
\hline Combined procedure & $23(57.5)$ & $28(56)$ & 0.887 \\
\hline $\begin{array}{l}\text { Mean } \mathrm{CHA}_{2} \mathrm{DS}_{2} \text {-VASc } \\
\text { score }\end{array}$ & $4.2 \pm 1.5$ & $4.1 \pm 1.3$ & 0.583 \\
\hline $\begin{array}{l}\text { Mean HAS-BLED } \\
\text { score }\end{array}$ & $2.3 \pm 0.8$ & $2.8 \pm 1.1$ & 0.010 \\
\hline Previous stoke/TIA & $21(52.5)$ & $32(64)$ & 0.271 \\
\hline Hypertension & $29(72.5)$ & $41(81)$ & 0.281 \\
\hline Diabetes mellitus & $9(22.5)$ & $12(24)$ & 0.555 \\
\hline $\begin{array}{l}\text { CAD Echocardiographic } \\
\text { characteristics }\end{array}$ & $7(17.5)$ & $11(22)$ & 0.596 \\
\hline $\mathrm{LAD}, \mathrm{mm}$ & $48.0 \pm 6.7$ & $49.6 \pm 5.7$ & 0.246 \\
\hline LVEDD, mm & $47.5 \pm 5.9$ & $49.7 \pm 6.0$ & 0.092 \\
\hline LVEF, $\%$ & $63.3 \pm 12.8$ & $63.9 \pm 7.8$ & 0.783 \\
\hline LAAC indication & LAAC indication & LAAC indication & LAAC indication \\
\hline Prior bleeding on OAT & $19(47.5)$ & $22(44)$ & 0.740 \\
\hline Stroke despite OAT & $3(7.5)$ & $5(10)$ & 0.967 \\
\hline Patient declining OAT & $12(30)$ & $17(34)$ & 0.687 \\
\hline others & $6(15)$ & $6(12)$ & 0.677 \\
\hline
\end{tabular}


Values are mean $\pm \mathrm{SD}$ or $\mathrm{n}(\%)$. The $\mathrm{P}$ values are results for the t-test or the chi-square test. BMI= body mass index, $\mathrm{PAF}=$ paroxysmal atrial fibrillation; $\mathrm{CAD}=$ coronary artery disease; $\mathrm{LAD}=$ left atrial diameter; $\mathrm{LVEDD}=$ left ventricular end-diastolic dimension; $\mathrm{LVEF}=$ left ventricular ejection fraction; OAT $=$ oral anticoagulant therapy.

\begin{tabular}{lll}
\hline Table 2 LAAC procedural data & Table 2 LAAC procedural data & Table 2 L $)$ \\
\hline & ICE $(\mathrm{n}=40)$ & TEE $(\mathrm{n}=50(100)$ \\
Success rate & $40(100)$ & LAA diame \\
LAA diameters & LAA diameters & $27.52 \pm 4.1$ \\
LAA orifice, mm & $27.65 \pm 3.21$ & $22.90 \pm 4.0$ \\
Landing zone, mm & $22.83 \pm 2.69$ & Size of LAn \\
Size of LAmbre device & Size of LAmbre device & $1(2)$ \\
18-24 mm & $1(2.5)$ & $1(2)$ \\
$20-32 \mathrm{~mm}$ & $2(5)$ & $4(8)$ \\
$22-28 \mathrm{~mm}$ & $2(5)$ & $8(16)$ \\
$22-34 \mathrm{~mm}$ & $0(0)$ & $9(18)$ \\
$24-30 \mathrm{~mm}$ & $8(20)$ & $1(2)$ \\
$24-36 \mathrm{~mm}$ & $1(2.5)$ & $9(18)$ \\
$26-32 \mathrm{~mm}$ & $9(22.5)$ & $3(6)$ \\
$26-38 \mathrm{~mm}$ & $3(7.5)$ & $4(8)$ \\
$28-34 \mathrm{~mm}$ & $6(15)$ & $3(6)$ \\
$30-36 \mathrm{~mm}$ & $4(10)$ & $3(6)$ \\
$32-36 \mathrm{~mm}$ & $2(5)$ & $2(4)$ \\
$34-38 \mathrm{~mm}$ & $1(2.5)$ & $2(4)$ \\
$36-40 \mathrm{~mm}$ & $1(2.5)$ & $710.44 \pm 2$ \\
Fluoroscopy time, sec & $286.30 \pm 197.07$ & $372.52 \pm 2$ \\
Radiation dose, mGy & $104.85 \pm 100.85$ & $180.40 \pm 10$ \\
Contrast usage, ml & $59.25 \pm 28.79$ & $61.28 \pm 22$. \\
Procedure time, min & $54.45 \pm 17.94$ & $18(36)$ \\
Unable to show long-axis view of the device at least at one angle & 0 & $25(50)$ \\
Devices without recapture & $22(55)$ & $11(22)$ \\
Resizing of the device & $8(20)$ & Peri-device \\
Peri-device leak post release on ICE/TEE & Peri-device leak post release on ICE/TEE \\
None & $38(95)$ & $47(94)$
\end{tabular}

Values are mean $\pm \mathrm{SD}, \mathrm{n}(\%)$

$\mathrm{LAA}=$ left atrial appendage.

Table 3 Residual flow and residual ASDs at 45-day TEE follow-up Table 3 Residual flow and residual ASDs at 45-day

Complete sealing rate

$\operatorname{ICE}(\mathrm{n}=38)$

$<1 \mathrm{~mm}$ leak $26(68.4)$

$1-3 \mathrm{~mm}$ leak 0

$>3 \mathrm{~mm}$ leak 12

Residual ASD

0

ASD size 
Values are $\mathrm{n}(\%), \mathrm{n} / \mathrm{N}(\%)$

$\mathrm{ASD}=$ atrial septal defect. 